\title{
On the existence of fuzzy solutions for partial hyperbolic functional differential equations
}

\author{
Hoang Viet Long ${ }^{1}$, Nguyen Thi Kim Son, ${ }^{2}$ Ha Thi Thanh Tam, ${ }^{3}$ Bui Cong Cuong ${ }^{4}$ \\ ${ }^{1}$ Department of Basic Science, University of Transport and Communications, ${ }^{\dagger}$ \\ Lang Thuong, Dong Da, Hanoi, Vietnam \\ ${ }^{2}$ Department of Mathematics, Hanoi University of Education \\ 136 Xuan Thuy, Cau Giay, Hanoi, Vietnam \\ ${ }^{3}$ Department of Mathematics, Hanoi University of Education \\ 136 Xuan Thuy, Hanoi, Vietnam \\ ${ }^{4}$ Institute of Mathematics, Vietnamese Academy of Science and Technology \\ 18 Hoang Quoc Viet Road, 10307 Hanoi, Vietnam
}

Received 25 August 2013

Accepted 11 May 2014

\begin{abstract}
In this paper, we consider the boundary valued problems for fuzzy partial hyperbolic functional differential equations with local and integral boundary conditions. A new weighted metric is used to investigate the existence and uniqueness of fuzzy solutions for these problems in a complete fuzzy metric space. Our results are demonstrated in some numerical examples in which we use the same strategy as BuckleyFeuring to build fuzzy solutions from fuzzifying the deterministic solutions. Then by using the continuity of the Zadeh's extension principle combining with numerical simulations for $\alpha$-cuts of fuzzy solutions, we give some representations of the surfaces of fuzzy solutions.
\end{abstract}

Keywords: Partial hyperbolic functional differential equations, fuzzy solution, local condition, boundary condition, fixed point, Zadeh's extension principle.

\section{Introduction}

The rise and development of science and technology such as computer science, robotics, artificial intelligence, language theory, etc, force us to be engaged in specifying imprecise notions. In 1965, Zadeh began to study fuzzy set theory ${ }^{1}$, which is a tool that makes possible the description of vague notions. Today, fuzzy set theory has become a fashionable theory used in many branches of real life such as dynamics, computer, biological phenomena, financial forecasting, etc. The concepts of fuzzy sets, fuzzy numbers, fuzzy metric spaces, fuzzy valued functions and necessary calculus of fuzzy functions were introduced $\mathrm{in}^{2,3}$. The fuzzy derivative was first investigated by Chang and Zadeh in ${ }^{4}$, later Dubois and Prade ${ }^{5}$ defined the fuzzy derivative by using Zadeh's extension principle and was followed by Puri and Ralescu ${ }^{6}$. In view of the development of calculus for fuzzy functions, the investigation of fuzzy differential equations (fuzzy DEs)

*Corresponding author: hvlong@utc.edu.vn

${ }^{\dagger}$ This work was financially supported by NAFOSTED - Vietnam under contract No.101.02-2014.33. 
and fuzzy partial differential equations (fuzzy PDEs) have been initiated ${ }^{7,8,9}$.

In recent years, there has been a significant development in fuzzy calculus techniques in fuzzy DEs and fuzzy differential inclusions, some recent contributions can be seen for example in the papers of Chalco - Cano and Román - Flores ${ }^{10}$, Choudary and Donchev ${ }^{11}$, Li et al. ${ }^{12}$, Rodríguez-López ${ }^{13}$, or the monograph of Lakshmikantham and Mohapatra ${ }^{3}$ and the references cited therein. However, the development of fuzzy PDEs has not been commensurate with its importance. Since Buckley and Feuring gained the existence of BF solutions and Seikkala solutions for fuzzy PDEs by crisp solution and the extension principle ${ }^{7}$, some other efforts have been done to deal with this kind of equations and the achievements are included in some researches of Allahviranloo et al. ${ }^{14}$, Arara et al. ${ }^{15}$, Bertone et al. ${ }^{16}$, Long et al. ${ }^{17}$ and Chen et al. ${ }^{18}$. Especially, in ${ }^{14}$ Allahviranloo and his coworkers succeeded in applying the same strategy as Buckley-Feuring in ${ }^{7}$ to find the exact solutions for fuzzy wave-like equations with variable coefficients. In our understanding, this results can be considered as the significant contributions on fuzzy linear PDEs.

Functional differential equations with statedependent delay appear frequently in applications as model of equations and for this reason the study of this type of equations has received great attention in the last year; see for instance ${ }^{19}$ and the references therein. The literatures related to partial functional differential equations with state-dependent delay are limited; see for instance ${ }^{20,21}$.

In this paper we are interested by the existence of fuzzy solutions of some boundary valued problems for partial hyperbolic functional differential equations (PHFDEs) with state-dependent delay. Our results may be interpreted as extensions of previous results of Arara et al. ${ }^{15}$ for fuzzy hyperbolic PDEs with local and nonlocal initial conditions and Bertone et al. ${ }^{16}$ with linear type of hyperbolic equations. The results in ${ }^{15}$ based on some complicated constraints on data and domain. Meanwhile, those conditions may be not necessary or can be reduced to be milder. In this paper, new weighted metrics are used and suitable weighted numbers are chosen in order to prove that the existence and uniqueness of fuzzy solutions only depend on the Lipschitz property of the right side of the equations. Especially, we investigate the well-posedness for fuzzy boundary value problems with integral boundary conditions. This class of equations has various applications in applied fields such as blood flow problems, chemical engineering, thermoplasticity, underground water flow, population dynamics, and so forth. For a detailed description of the integral boundary conditions, we refer to the papers ${ }^{22}$. Moreover, we know that fuzzy boundary value problems with integral boundary conditions constitute a very interesting and important class of problems. They include two, three, multi-points boundary value problems and local, nonlocal initial conditions problems as the special cases. Therefore the results of the present paper can be considered as a contribution to the subject.

The remainder of the paper is arranged as follows. In section 2, we give some basic preliminaries which will be used throughout this paper. Section 3 and 4 give the main results of the existence and uniqueness of fuzzy solutions for some class of PHFDEs with local conditions and integral conditions. Here the idea of a new weighted metric is used to get the best conditions on databases to ensure the existence and uniqueness of solutions. Especially in section 5, some illustrated example for our results are given with some numerical simulation for $\alpha$-cuts of the fuzzy solutions.

\section{Preliminaries}

In this section, we recall some concepts of fuzzy metric space that will be used throughout the paper. For a more thorough treatise on fuzzy analysis, we refer to the books ${ }^{2,3}$.

We denote the set consisting of all nonempty compact, convex subsets of $\mathbb{R}^{n}$ by $\mathscr{K}_{C}^{n}$. Let $A$ and $B$ be two nonempty bounded subsets of $\mathscr{K}_{C}^{n}$. Denote by $\|$.$\| a norm in \mathbb{R}^{n}$. The distance between $A$ and $B$ is defined by the Hausdorff metric

$d_{H}(A, B)=\max \left\{\sup _{a \in A} \inf _{b \in B}\|a-b\|, \sup _{b \in B} \inf _{a \in A}\|a-b\|\right\}$, then $\left(\mathscr{K}_{C}^{n}, d_{H}\right)$ is a complete metric space. 
Let $E^{n}$ be the space of functions $u: \mathbb{R}^{n} \rightarrow[0,1]$ satisfying

i) there exists a $x_{0} \in \mathbb{R}^{n}$ such that $u\left(x_{0}\right)=1$;

ii) $u$ is fuzzy convex, that is for $x, y \in E^{n}$ and $0 \leqslant \lambda \leqslant 1$,

$$
u(\lambda x+(1-\lambda) y) \geqslant \min [u(x), u(y)]
$$

iii) $u$ is semi-continuous;

iv) $[u]^{0}=\overline{\left\{x \in \mathbb{R}^{n}: u(x)>0\right\}}$ is a compact set in $\mathbb{R}^{n}$.

We call $u \in E^{n}$ a fuzzy number and the $\alpha$-cuts or level sets of $u$ are defined by

$$
[u]^{\alpha}=\left\{x \in \mathbb{R}^{n}: u(x) \geqslant \alpha\right\} \text { for each } 0<\alpha \leqslant 1 .
$$

Then from $(i)$ to $(i v)$, it follows that $[u]^{\alpha}$ is in $\mathscr{K}_{C}^{n}$.

The $\alpha$-cut of fuzzy number can be presented by an order pair of function $\left[u_{1}(\alpha), u_{2}(\alpha)\right], 0<\alpha \leqslant 1$, called parametric form, which satisfies the following requirements

- $u_{1}(\alpha)$ is a bounded left continuous nondecreasing function of $\alpha$,

- $u_{2}(\alpha)$ is a bounded left continuous non-increasing function of $\alpha$,

- $u_{1}(1)=u_{2}(1)$.

If $g: \mathbb{R}^{n} \times \mathbb{R}^{n} \rightarrow \mathbb{R}^{n}$ is a function, then according to Zadeh's extension principle we can extend $g$ to $E^{n} \times E^{n} \rightarrow E^{n}$ by the function defined by

$$
g(u, v)(t)=\sup _{t=g(x, y)} \min \{u(x), v(y)\} .
$$

If $g$ is continuous then the continuity property of extension principle states that

$$
[g(u, v)]^{\alpha}=g\left([u]^{\alpha},[v]^{\alpha}\right)
$$

for all $u, v \in \mathbb{E}^{n}, 0 \leqslant \alpha \leqslant 1$.

Especially, we will define addition and scalar multiplication of fuzzy numbers in $E^{n}$ levelsetwise, that is, for all $u, v \in E^{n}, 0 \leqslant \alpha \leqslant 1, k \in \mathbb{R} \backslash\{0\}$,

$$
[u+v]^{\alpha}=[u]^{\alpha}+[v]^{\alpha}
$$

and

$$
[k u]^{\alpha}=k[u]^{\alpha},
$$

where

$$
(u+v)(t)=\sup _{t=t_{1}+t_{2}} \min \left\{u\left(t_{1}\right), v\left(t_{2}\right)\right\}
$$

and

$$
k u(t)=u(t / k) .
$$

Supremum metric is the most commonly used metric on $E^{n}$ defined by the Hausdorff metric distance between the level sets of the fuzzy numbers

$$
d_{\infty}(u, v)=\sup _{0<\alpha \leqslant 1} H_{d}\left([u]^{\alpha},[v]^{\alpha}\right)
$$

for all $u, v \in E^{n}$. It is obviously that $\left(E^{n}, d_{\infty}\right)$ is a complete metric space. From the properties of Hausdorff metric, we infer

- $d_{\infty}(c u, c v)=|c| d_{\infty}(u, v)$,

- $d_{\infty}\left(u+u^{\prime}, v+v^{\prime}\right) \leqslant d_{\infty}(u, v)+d_{\infty}\left(u^{\prime}, v^{\prime}\right)$,

- $d_{\infty}(u+w, v+w)=d_{\infty}(u, v)$,

for all $u, v, u^{\prime}, v^{\prime}, w \in E^{n}$ and $c \in \mathbb{R} \backslash\{0\}$.

For any positive real number $r>0$, we denote $J_{0}=[-r, 0] \times[-r, 0], \quad J_{r}=[-r, a] \times[-r, b], \widetilde{J}_{r}=$ $J_{r} \backslash(0, a] \times(0, b]$ and $J_{a b}=[0, a] \times[0, b]$.

Definition 1. A mapping $f: J_{r} \rightarrow E^{n}$ is called continuous at $\left(t_{0}, s_{0}\right) \in J_{r}$ if the multi-valued mapping $f_{\alpha}(t, s)=[f(t, s)]^{\alpha}$ is continuous at $\left(t_{0}, s_{0}\right)$ with respect to the Hausdorff metric $d_{H}$ for all $\alpha \in[0,1]$.

In this paper, we denote by $C\left(J_{r}, E^{n}\right)$ the space of all continuous functions $f: J_{r} \rightarrow E^{n}$ with the supremum weighted metric $H_{\lambda}$ defined by

$$
H_{\lambda}(f, g)=\sup _{(t, s) \in J_{r}}\left\{d_{\infty}(f(t, s), g(t, s)) e^{-\lambda(t+s)}\right\} .
$$

Since $\left(E^{n}, d_{\infty}\right)$ is a complete metric space, it can be shown that $\left(C\left(J_{r}, E^{n}\right), H_{\lambda}\right)$ is also a complete metric space for $\lambda>0$ arbitrary.

Definition 2. A mapping $f: J_{r} \times E^{n} \rightarrow E^{n}$ is called continuous at point $\left(t_{0}, s_{0}, u_{0}\right) \in J_{r} \times E^{n}$ provided that for any fixed $\alpha \in[0,1]$ and arbitrary $\varepsilon>0$, there exists $\delta(\varepsilon, \alpha)>0$ such that

$$
d_{H}\left([f(t, s, u)]^{\alpha},\left[f\left(t_{0}, s_{0}, u_{0}\right)\right]^{\alpha}\right)<\varepsilon
$$


whenever $(t, s, u) \in J_{r} \times E^{n}$ satisfying

$\max \left\{\left|t-t_{0}\right|,\left|s-s_{0}\right|, d_{H}\left([u]^{\alpha},\left[u_{0}\right]^{\alpha}\right)\right\}<\delta(\varepsilon, \alpha)$.

Definition 3. For any fuzzy valued mapping $f$ : $J_{a b} \rightarrow E^{n}$, the integral of $f$ over $J_{a b}$, denoted by $\int_{0}^{a} \int_{0}^{b} f(t, s) d s d t$, is defined by

$\left[\int_{0}^{a} \int_{0}^{b} f(t, s) d s d t\right]^{\alpha}=\int_{0}^{a} \int_{0}^{b}[f(t, s)]^{\alpha} d s d t$ $=\left\{\int_{0}^{a} \int_{0}^{b} v(t, s) d s d t \mid v: J_{a b} \rightarrow \mathbb{R}^{n}\right.$ is a measurable selection for $\left.[f(t, s)]^{\alpha}\right\}$,

for all $\alpha \in(0,1]$. A function $f: J_{a b} \rightarrow E^{n}$ is integrable on $J_{a b}$ if $\int_{0}^{a} \int_{0}^{b} f(t, s) d s d t$ is in $E^{n}$.

Let $f, g: J_{a b} \rightarrow E^{n}$ be integrable and $\lambda \in \mathbb{R}$. The integral has the elementary properties as follows

- $\int_{0}^{a} \int_{0}^{b}[f(t, s)+g(t, s)] d s d t=\int_{0}^{a} \int_{0}^{b} f(t, s) d s d t$ $+\int_{0}^{a} \int_{0}^{b} g(t, s) d s d t$

- $\int_{0}^{a} \int_{0}^{b} \lambda f(t, s) d s d t=\lambda \int_{0}^{a} \int_{0}^{b} f(t, s) d s d t$,

- $d_{\infty}\left(\int_{0}^{a} \int_{0}^{b} f(t, s) d s d t, \int_{0}^{a} \int_{0}^{b}(t, s) d s d t\right)$ $\leqslant \int_{0}^{a} \int_{0}^{b} d_{\infty}(f(t, s), g(t, s)) d s d t$.

Definition 4. Let $x, y \in E^{n}$. If there exists $z \in E^{n}$ such that $x=y+z$ then we call $z$ the Hukuharadifference of $x$ and $y$, denoted by $x-y$.

Definition 5. For any fuzzy valued mapping $f$ : $J_{a b} \rightarrow E^{n}$, the fuzzy partial derivative of $f$ with respect to $x$ at the point $\left(x_{0}, y_{0}\right) \in J$ is a fuzzy set $\frac{\partial f\left(x_{0}, y_{0}\right)}{\partial x} \in E^{n}$ which is defined by

$$
\frac{\partial f\left(x_{0}, y_{0}\right)}{\partial x}=\lim _{h \rightarrow 0} \frac{f\left(x_{0}+h, y_{0}\right)-f\left(x_{0}, y_{0}\right)}{h} .
$$

Here the limit is taken in the metric space $\left(E^{n}, d_{\infty}\right)$ and $u-v$ is the Hukuhara-difference of $u$ and $v$ in $E^{n}$. The fuzzy partial derivatives of $f$ with respect to $y$ and higher order of fuzzy partial derivative of $f$ at the point $\left(x_{0}, y_{0}\right) \in J_{a b}$ are defined similarly.

\section{Fuzzy partial hyperbolic functional differential equations with local conditions}

In the first part of this section, we consider the following fuzzy PHFDEs

$$
\frac{\partial^{2} u(x, y)}{\partial x \partial y}=f\left(x, y, u_{(x, y)}\right), \quad(x, y) \in J_{a b}
$$

with initial condition

$$
u(x, y)=\varphi(x, y),(x, y) \in \widetilde{J_{r}}
$$

and local conditions are

$$
\left\{\begin{array}{l}
u(x, 0)=\eta_{1}(x), x \in[0, a] \\
u(0, y)=\eta_{2}(y), y \in[0, b] \\
\varphi(0,0)=\eta_{1}(0)=\eta_{2}(0)
\end{array}\right.
$$

where $f: J_{a b} \times C\left(J_{0}, E^{n}\right) \rightarrow E^{n}$ is a continuous function, $\eta_{1} \in C\left([0, a], E^{n}\right), \eta_{2} \in C\left([0, b], E^{n}\right)$ and $\varphi \in$ $C\left(J_{0}, E^{n}\right)$ are given functions and state-dependent delays $u_{(x, y)}(t, s)$ is defined by

$$
u_{(x, y)}(t, s)=u(x+t, y+s),(t, s) \in J_{0},
$$

here, $u_{(x, y)}(.,$.$) represents the history of the state$ from time $(x-r, y-r)$ up to the present time $(x, y)$. Hypothesis (H). There exists $K>0$ such that

$$
\begin{aligned}
& d_{\infty}\left(f\left(x, y, u_{(x, y)}\right), f\left(x, y, v_{(x, y)}\right)\right) \\
& \quad \leqslant K d_{\infty}(u(x+\omega, y+\theta), v(x+\omega, y+\theta))
\end{aligned}
$$

holds for all $u, v \in C\left(J_{r}, E^{n}\right), \quad(x, y) \in J_{a b}$ and $(\omega, \theta) \in J_{0}$.

Definition 6. A function $u \in C\left(J_{r}, E^{n}\right)$ is called a fuzzy solution of the problem (1)-(3) if it satisfies

$$
\begin{aligned}
u(x, y)= & \eta_{1}(x)+\eta_{2}(y)-\varphi(0,0)+ \\
& \int_{0}^{x} \int_{0}^{y} f\left(t, s, u_{(t, s)}\right) d s d t, \quad(x, y) \in J_{a b} \\
u(x, y)= & \eta_{1}(x)+\eta_{2}(y)-\varphi(0,0)+ \\
& \int_{0}^{x} \int_{0}^{y} f\left(t, s, u_{(t, s)}\right) d s d t, \quad(x, y) \in J_{a b}
\end{aligned}
$$

and $u(x, y)=\varphi(x, y),(x, y) \in \widetilde{J_{r}}$. By using the weighted metric $H_{\lambda}$ in the space $C\left(J_{r}, E^{n}\right)$, we 
receive following result about the existence and uniqueness of solutions of the problem (1)-(3).

Theorem 1. Assume that the Hypothesis $(H)$ is satisfied. Then for all $\lambda>\sqrt{K}$, the problem (1)-(3) has a unique solution in $C\left(J_{r}, E^{n}\right)$ with metric $H_{\lambda}$. Proof. From the Definition 6, we realize that fuzzy solution of the problem (1)-(3) (if it exists) is a fixed point of the operator $N_{1}: C\left(J_{r}, E^{n}\right) \rightarrow C\left(J_{r}, E^{n}\right)$ defined as

$N_{1}(u(x, y))= \begin{cases}\eta_{1}(x)+\eta_{2}(y)-\varphi(0,0) & \\ +\int_{0}^{x} \int_{0}^{y} f\left(t, s, u_{(t, s)}\right) d s d t & (x, y) \in J_{a b} \\ \varphi(x, y) & (x, y) \in \widetilde{J}_{r} .\end{cases}$

We will show that $N_{1}$ is a contraction operator.

Indeed, for arbitrary $u, v \in C\left(J_{r}, E^{n}\right)$, and $(x, y) \in$ $J_{a b}$ then

$$
\begin{aligned}
N_{1}(u(x, y))= & \eta_{1}(x)+\eta_{2}(y)-\varphi(0,0) \\
& +\int_{0}^{x} \int_{0}^{y} f\left(t, s, u_{(t, s)}\right) d s d t
\end{aligned}
$$

and

$$
\begin{aligned}
N_{1}(v(x, y))= & \eta_{1}(x)+\eta_{2}(y)-\varphi(0,0) \\
& +\int_{0}^{x} \int_{0}^{y} f\left(t, s, v_{(t, s)}\right) d s d t .
\end{aligned}
$$

From the properties of supremum metric, we have following inequality

$$
\begin{aligned}
& d_{\infty}\left(N_{1}(u(x, y)), N_{1}(v(x, y))\right) \\
& \leqslant d_{\infty}\left(\int_{0}^{x} \int_{0}^{y} f\left(t, s, u_{(t, s)}\right) d s d t,\right. \\
& \left.\int_{0}^{x} \int_{0}^{y} f\left(t, s, v_{(t, s)}\right) d s d t\right) \\
& \leqslant \int_{0}^{x} \int_{0}^{y} d_{\infty}\left(f\left(t, s, u_{(t, s)}\right), f\left(t, s, v_{(t, s)}\right)\right) d s d t \\
& \leqslant \int_{0}^{x} \int_{0}^{y} K d_{\infty}(u(t+\omega, s+\theta), v(t+\omega, s+\theta)) d s d t \\
& \leqslant K H_{\lambda}(u, v) \int_{0}^{x} \int_{0}^{y} e^{\lambda(\omega+\theta+t+s)} d s d t \\
& \leqslant K H_{\lambda}(u, v) \frac{1}{\lambda} e^{\lambda(\omega+\theta)} \int_{0}^{x}\left(e^{\lambda(t+y)}-e^{\lambda t}\right) d t \\
& \leqslant \frac{K}{\lambda^{2}} H_{\lambda}(u, v) e^{\lambda(\omega+\theta)}\left(e^{\lambda x}-1\right)\left(e^{\lambda y}-1\right) .
\end{aligned}
$$

Multiplying both sides of this inequality by $e^{-\lambda(x+y)}$, then taking supremum metric we receive

$$
\begin{aligned}
& e^{-\lambda(x+y)} d_{\infty}\left(N_{1}(u(x, y)), N_{1}(v(x, y))\right) \\
& \leqslant \frac{K}{\lambda^{2}} H_{\lambda}(u, v) e^{\lambda(\omega+\theta)}\left(e^{\lambda x}-1\right)\left(e^{\lambda y}-1\right) e^{-\lambda(x+y)} \\
& \leqslant \frac{K}{\lambda^{2}} H_{\lambda}(u, v) e^{\lambda(\omega+\theta)} e^{\lambda x} e^{\lambda y} e^{-\lambda(x+y)} \\
& \leqslant \frac{K}{\lambda^{2}} H_{\lambda}(u, v) . \\
& \quad \text { If }(x, y) \in \widetilde{J}_{r} \text { then } \\
& \quad d_{\infty}\left(N_{1}(u(x, y)), N_{1}(v(x, y))\right)=0 .
\end{aligned}
$$

Thus

$$
e^{-\lambda(x+y)} d_{\infty}\left(N_{1}(u(x, y)), N_{1}(v(x, y))\right) \leqslant \frac{K}{\lambda^{2}} H_{\lambda}(u, v)
$$

holds for all $(x, y) \in J_{r}$. From the definition of metric $H_{\lambda}$ we have

$$
H_{\lambda}\left(N_{1}(u), N_{1}(v)\right) \leqslant \frac{K}{\lambda^{2}} H_{\lambda}(u, v),
$$

for all $u, v \in C\left(J_{r}, E^{n}\right)$.

So by choosing $\lambda>\sqrt{K}$, we imply that $N_{1}$ is a contraction operator and by fixed point theorem, $N_{1}$ has a unique fixed point, that is a solution of the problem (1)-(3). The proof is completed.

In this second part, we consider the fuzzy PHFDEs in more general form

$$
\frac{\partial^{2} u(x, y)}{\partial x \partial y}=\frac{\partial(p(x, y) u(x, y))}{\partial y}+f\left(x, y, u_{(x, y)}\right),
$$

for $(x, y) \in J_{a b}$, with the initial condition is

$$
u(x, y)=\varphi(x, y),(x, y) \in \widetilde{J}_{r}
$$

and the local conditions are

$$
\left\{\begin{array}{l}
u(x, 0)=\eta_{1}(x), x \in[0, a], \\
u(0, y)=\eta_{2}(y), y \in[0, b], \\
\varphi(0,0)=\eta_{1}(0)=\eta_{2}(0),
\end{array}\right.
$$

where $f: J_{a b} \times C\left(J_{0}, E^{n}\right) \rightarrow E^{n}, p \in C\left(J_{a b}, \mathbb{R}\right), \eta_{1} \in$ $C\left([0, a], E^{n}\right), \eta_{2} \in C\left([0, b], E^{n}\right)$ are given functions and $\varphi \in C\left(J_{0}, E^{n}\right)$. 
Definition 7. A function $u \in C\left(J_{r}, E^{n}\right)$ is called a solution of the problem (5)-(7) if it satisfies

$$
\begin{aligned}
u(x, y)= & q(x, y)+\int_{0}^{x} p(t, y) u(t, y) d t \\
& +\int_{0}^{x} \int_{0}^{y} f\left(t, s, u_{(t, s)}\right) d s d t
\end{aligned}
$$

where

$$
\begin{aligned}
q(x, y)= & \eta_{1}(x)+\eta_{2}(y)-\varphi(0,0) \\
& -\int_{0}^{x} p(t, 0) \eta_{1}(t) d t,(x, y) \in J_{a b}
\end{aligned}
$$

and

$$
u(x, y)=\varphi(x, y),(x, y) \in \widetilde{J}_{r} .
$$

Theorem 2. Assume that the hypothesis $(H)$ is satisfied. Then for all $\lambda>0$ satisfying

$$
\lambda^{2}-\sup _{(t, s) \in J_{a b}}|p(t, s)| \lambda-K>0
$$

the problem (5)-(7) has a unique solution in the metric space $C\left(J_{r}, E^{n}\right)$ with metric $H_{\lambda}$.

Proof. Transform the problem (5)-(7) into a fixed point problem. To this end, we consider the operator $N_{2}: C\left(J_{r}, E^{n}\right) \rightarrow C\left(J_{r}, E^{n}\right)$ defined by

$N_{2}(u(x, y))=\left\{\begin{array}{l}q(x, y)+\int_{0}^{x} p(t, y) u(t, y) d t \\ +\int_{0}^{x} \int_{0}^{y} f\left(t, s, u_{(t, s)}\right) d s d t,(x, y) \in J_{a b}, \\ \varphi(x, y),(x, y) \in \widetilde{J_{r}},\end{array}\right.$

where

$q(x, y)=\eta_{1}(x)+\eta_{2}(y)-\varphi(0,0)-\int_{0}^{x} p(t, 0) \eta_{1}(t) d t$.

In order to show $N_{2}$ being a contraction operator, we consider $u, v \in C\left(J_{r}, E^{n}\right)$ and $(x, y) \in J_{a b}$. We have

$$
\begin{gathered}
d_{\infty}\left(N_{2}(u(x, y)), N_{2}(v(x, y))\right) \\
\leqslant d_{\infty}\left(\int_{0}^{x} p(t, y) u(t, y) d t, \int_{0}^{x} p(t, y) v(t, y) d t\right) \\
+d_{\infty}\left(\int_{0}^{x} \int_{0}^{y} f\left(t, s, u_{(t, s)}\right) d s d t\right. \\
\left.\int_{0}^{x} \int_{0}^{y} f\left(t, s, v_{(t, s)}\right) d s d t\right) .
\end{gathered}
$$

Set $p=\sup _{(t, s) \in J_{a b}}|p(t, s)|$. One gets following assessment

$$
\begin{aligned}
& d_{\infty}\left(\int_{0}^{x} p(t, y) u(t, y) d t, \int_{0}^{x} p(t, y) v(t, y) d t\right) \\
& \quad \leqslant \sup _{(t, s) \in J_{a b}}|p(t, s)| \int_{0}^{x} d_{\infty}(u(t, y), v(t, y)) d t \\
& \quad \leqslant p \int_{0}^{x} d_{\infty}(u(t, y), v(t, y)) d t .
\end{aligned}
$$

By doing the same previous arguments one gets

$$
\begin{aligned}
e^{-\lambda(x+y)} & d_{\infty}\left(\int_{0}^{x} p(t, y) u(t, y) d t, \int_{0}^{x} p(t, y) v(t, y) d t\right) \\
& \leqslant p e^{-\lambda(x+y)} \int_{0}^{x} d_{\infty}(u(t, y), v(t, y)) d t \\
& \leqslant p H_{\lambda}(u, v) e^{-\lambda(x+y)} \int_{0}^{x} e^{\lambda(t+y)} d t \\
& \leqslant p H_{\lambda}(u, v) e^{-\lambda(x+y)} \frac{1}{\lambda}\left[e^{\lambda(x+y)}-e^{\lambda y}\right] \\
& \leqslant \frac{p}{\lambda} H_{\lambda}(u, v) .
\end{aligned}
$$

The second term in the right side of (9) is estimated as the same in inequality (4), that leads to

$$
\begin{gathered}
d_{\infty}\left(\int_{0}^{x} \int_{0}^{y} f\left(t, s, u_{(t, s)}\right) d s d t, \int_{0}^{x} \int_{0}^{y} f\left(t, s, v_{(t, s)}\right) d s d t\right) \\
\leqslant \frac{K}{\lambda^{2}} H_{\lambda}(u, v) e^{\lambda(\omega+\theta)}\left(e^{\lambda x}-1\right)\left(e^{\lambda y}-1\right)
\end{gathered}
$$

and

$$
\begin{aligned}
e^{-\lambda(x+y)} & d_{\infty}\left(\int_{0}^{x} \int_{0}^{y} f\left(t, s, u_{(t, s)}\right) d s d t\right. \\
& \left.\int_{0}^{x} \int_{0}^{y} f\left(t, s, v_{(t, s)}\right) d s d t\right) \\
& \leqslant \frac{K}{\lambda^{2}} H_{\lambda}(u, v) .
\end{aligned}
$$

When $(x, y) \in \widetilde{J_{r}}$

$$
H_{\lambda}\left(N_{2}(u(x, y)), N_{2}(v(x, y))\right)=0 .
$$

From (9) to (12) we can see that

$$
e^{-\lambda(x+y)} d_{\infty}\left(N_{2}(u), N_{2}(v)\right) \leqslant\left(\frac{p}{\lambda}+\frac{K}{\lambda^{2}}\right) H_{\lambda}(u, v)
$$


satisfies for every $(x, y) \in J_{r}$.

Hence

$$
H_{\lambda}\left(N_{2}(u), N_{2}(v)\right) \leqslant\left(\frac{p}{\lambda}+\frac{K}{\lambda^{2}}\right) H_{\lambda}(u, v),
$$

for all $u, v \in C\left(J_{r}, E^{n}\right)$.

It is easy to see that for all $\lambda>0$ satisfying (8) we have $\frac{p}{\lambda}+\frac{K}{\lambda^{2}}<1$. It follows that $N_{2}$ is a contraction operator. Therefore the problem (5)-(7) has a unique solution, which is the fixed point of the operator $\mathrm{N}_{2}$. The theorem is proven completely.

\section{Fuzzy partial hyperbolic functional differential equations with integral boundary conditions}

In this section, we consider the fuzzy PHFDEs

$$
\frac{\partial^{2} u(x, y)}{\partial x \partial y}=\frac{\partial(p(x, y) u(x, y))}{\partial y}+f\left(x, y, u_{(x, y)}\right),
$$

for $(x, y) \in J_{a b}$, with the following integral boundary conditions

$$
\begin{aligned}
& u(x, 0)+\int_{0}^{y} k_{1}(x) u(x, s) d s=g_{1}(x, y), \\
& u(0, y)+\int_{0}^{x} k_{2}(y) u(t, y) d t=g_{2}(x, y),
\end{aligned}
$$

for $(x, y) \in J_{a b}$ and

$$
u(x, y)=\varphi(x, y), \quad(x, y) \in \widetilde{J_{r}}
$$

where $f: J_{a b} \times C\left(J_{0}, E^{n}\right) \rightarrow E^{n}, p \in C\left(J_{a b}, \mathbb{R}\right)$ is a continuous function; $g_{1}, g_{2} \in C\left(J_{a b}, E^{n}\right), k_{1} \in$ $C([0, a], \mathbb{R}), k_{2} \in C([0, b], \mathbb{R})$ are given functions and $\varphi \in C\left(J_{0}, E^{n}\right)$ satisfied $g_{1}(x, 0)=\varphi(x, 0)$ and $g_{2}(0, y)=\varphi(0, y)$.

Definition 8. A function $u \in C\left(J_{r}, E^{n}\right)$ is called a solution of the problem (14)-(17) if $u(x, y)=\varphi(x, y)$ for $(x, y) \in \widetilde{J}_{r}$ and for $(x, y) \in J_{a b}$ it satisfies the fol- lowing integral equation

$$
\begin{aligned}
& u(x, y)=Q(x, y)-\int_{0}^{y} k_{1}(x) u(x, s) d s \\
& -\int_{0}^{x} k_{2}(y) u(t, y) d t-k_{1}(0) \int_{0}^{y} \int_{0}^{x} k_{2}(s) u(t, s) d t d s \\
& +\int_{0}^{x} p(t, y) u(t, y) d t-\int_{0}^{x} p(t, 0) u(t, 0) d t \\
& +\int_{0}^{x} \int_{0}^{y} f\left(t, s, u_{(t, s)}\right) d s d t
\end{aligned}
$$

where

$$
\begin{aligned}
Q(x, y)= & g_{1}(x, y)+g_{2}(x, y)-g_{1}(0, y) \\
& +k_{1}(0) \int_{0}^{y} g_{2}(x, s) d s .
\end{aligned}
$$

For simplicity, we set $p=\sup _{(t, s) \in J_{a b}}|p(t, s)|$, $k_{1}=\sup _{x \in[0, a]}\left|k_{1}(x)\right|$ and $k_{2}=\sup _{x \in[0, b]}\left|k_{2}(y)\right|$. Let $u, v \in C\left(J_{r}, E^{n}\right)$ and $(x, y) \in J_{a b}$.

Theorem 3. The problem (14)-(17) has a unique solution in $C\left(J_{r}, E^{n}\right)$ provided that the Hypothesis $(H)$ is satisfied and

$$
\frac{k_{1}+k_{2}+2 p}{\lambda}+\frac{k_{1} k_{2}+K}{\lambda^{2}}<1 .
$$

Proof. Consider operator

$$
N_{3}: C\left(J_{r}, E^{n}\right) \rightarrow C\left(J_{r}, E^{n}\right)
$$

defined by

$$
\begin{aligned}
& N_{3}(u(x, y))=Q(x, y)-\int_{0}^{y} k_{1}(x) u(x, s) d s \\
& \quad-\int_{0}^{x} k_{2}(y) u(t, y) d t-k_{1}(0) \int_{0}^{y} \int_{0}^{x} k_{2}(s) u(t, s) d t d s \\
& \quad+\int_{0}^{x} p(t, y) u(t, y) d t-\int_{0}^{x} p(t, 0) u(t, 0) d t \\
& \quad+\int_{0}^{x} \int_{0}^{y} f\left(t, s, u_{(t, s)}\right) d s d t
\end{aligned}
$$

for $(x, y) \in J_{a b}$, where

$$
\begin{aligned}
Q(x, y)= & g_{1}(x, y)+g_{2}(x, y)-g_{1}(0, y) \\
& +k_{1}(0) \int_{0}^{y} g_{2}(x, s) d s
\end{aligned}
$$

and

$$
N_{3}(u(x, y))=\varphi(x, y),(x, y) \in \widetilde{J}_{r} .
$$


We will prove that $N_{3}$ is a contraction operator. In fact

$$
\begin{aligned}
& d_{\infty}\left(N_{3}(u(x, y)), N_{3}(v(x, y))\right) \\
& \leqslant d_{\infty}\left(\int_{0}^{y} k_{1}(x) u(x, s) d s, \int_{0}^{y} k_{1}(x) v(x, s) d s\right) \\
& +d_{\infty}\left(\int_{0}^{x} k_{2}(y) u(t, y) d t, \int_{0}^{x} k_{2}(y) v(t, y) d t\right) \\
& +d_{\infty}\left(k_{1}(0) \int_{0}^{y} \int_{0}^{x} k_{2}(s) u(t, s) d t d s\right. \\
& +d_{\infty}\left(\int_{0}^{x} p(t, y) u(t, y) d t, \int_{0}^{y} p(t, y) v(t, y) d t\right) \\
& +d_{\infty}\left(\int_{0}^{x} p(t, 0) u(t, 0) d t, \int_{0}^{x} p(t, 0) v(t, 0) d t\right) \\
& +d_{\infty}\left(\int_{0}^{x} \int_{0}^{y} f\left(t, s, u_{(t, s)}\right) d s d t\right. \\
& \left.\quad \int_{0}^{x} \int_{0}^{y} f\left(t, s, v_{(t, s)}\right) d s d t\right) .
\end{aligned}
$$

First of all, we have

$$
\begin{aligned}
& e^{-\lambda(x+y)} d_{\infty}\left(\int_{0}^{y} k_{1}(x) u(x, s) d s, \int_{0}^{y} k_{1}(x) v(x, s) d s\right) \\
& \leqslant e^{-\lambda(x+y)} \sup _{x \in[0, a]}\left|k_{1}(x)\right| d_{\infty}\left(\int_{0}^{y} u(x, s) d s, \int_{0}^{y} v(x, s) d s\right) \\
& \leqslant k_{1} e^{-\lambda(x+y)} \int_{0}^{y} d_{\infty}(u(x, s), v(x, s)) e^{-\lambda(x+s)} e^{\lambda(x+s)} d s \\
& \leqslant k_{1} H_{\lambda}(u, v) e^{-\lambda(x+y)} \int_{0}^{y} e^{\lambda(x+s)} d s \\
& =\frac{k_{1}}{\lambda} H_{\lambda}(u, v) e^{-\lambda(x+y)}\left(e^{\lambda(x+y)}-e^{\lambda x}\right) \\
& \leqslant \frac{k_{1}}{\lambda} H_{\lambda}(u, v) .
\end{aligned}
$$

Similarly, we obtain

$$
\begin{aligned}
e^{-\lambda(x+y)} & d_{\infty}\left(\int_{0}^{x} k_{2}(y) u(t, y) d t, \int_{0}^{x} k_{2}(y) v(t, y) d t\right) \\
& \leqslant \frac{k_{2}}{\lambda} H_{\lambda}(u, v) .
\end{aligned}
$$

Nevertheless

$$
\begin{aligned}
& e^{-\lambda(x+y)} d_{\infty}\left(k_{1}(0) \int_{0}^{y} \int_{0}^{x} k_{2}(s) u(t, s) d t d s\right. \\
& \left.k_{1}(0) \int_{0}^{y} \int_{0}^{x} k_{2}(s) v(t, s) d t d s\right) \\
& \leqslant k_{1} k_{2} e^{-\lambda(x+y)} \int_{0}^{y} \int_{0}^{x} d_{\infty}(u(t, s), v(t, s)) d t d s \\
& \leqslant k_{1} k_{2} H_{\lambda}(u, v) e^{-\lambda(x+y)} \int_{0}^{y} \int_{0}^{x} e^{\lambda(t+s)} d t d s \\
& \leqslant \frac{k_{1} k_{2}}{\lambda^{2}} H_{\lambda}(u, v) e^{-\lambda(x+y)}\left(e^{\lambda x}-1\right)\left(e^{\lambda y}-1\right) \\
& \leqslant \frac{k_{1} k_{2}}{\lambda^{2}} H_{\lambda}(u, v) .
\end{aligned}
$$

From (10) we get

$$
\begin{aligned}
e^{-\lambda(x+y)} & d_{\infty}\left(\int_{0}^{x} p(t, y) u(t, y) d t, \int_{0}^{x} p(t, y) v(t, y) d t\right) \\
& \leqslant \frac{p}{\lambda} H_{\lambda}(u, v)
\end{aligned}
$$

and

$$
\begin{aligned}
e^{-\lambda(x+y)} & d_{\infty}\left(\int_{0}^{x} p(t, 0) u(t, 0) d t, \int_{0}^{x} p(t, 0) v(t, 0) d t\right) \\
& \leqslant \frac{p}{\lambda} H_{\lambda}(u, v) .
\end{aligned}
$$

Finally, from (11) we have

$$
\begin{aligned}
e^{-\lambda(x+y)} & d_{\infty}\left(\int_{0}^{x} \int_{0}^{y} f\left(t, s, u_{(t, s)}\right) d s d t\right. \\
& \left.\int_{0}^{x} \int_{0}^{y} f\left(t, s, v_{(t, s)}\right) d s d t\right) \\
& \leqslant \frac{K}{\lambda^{2}} H_{\lambda}(u, v)
\end{aligned}
$$

It is clear that if $(x, y) \in \widetilde{J}_{r}$ then

$$
d_{\infty}\left(N_{3}(u(x, y)), N_{3}(v(x, y))\right)=0 .
$$

Substitute (19)-(24) into (18) after multiplying both sides by $e^{-\lambda(x+y)}$ we have

$$
\begin{aligned}
e^{-\lambda(x+y)} & d_{\infty}\left(N_{3}(u(x, y)), N_{3}(v(x, y))\right) \\
& \leqslant\left(\frac{k_{1}+k_{2}+2 p}{\lambda}+\frac{k_{1} k_{2}+K}{\lambda^{2}}\right) H_{\lambda}(u, v)
\end{aligned}
$$


holds for all $u, v \in C\left(J_{r}, E^{n}\right)$ and $(x, y) \in J_{r}$. That leads to

$$
\begin{aligned}
& H_{\lambda}\left(N_{3}(u), N_{3}(v)\right) \\
& \quad \leqslant\left(\frac{k_{1}+k_{2}+2 p}{\lambda}+\frac{k_{1} k_{2}+K}{\lambda^{2}}\right) H_{\lambda}(u, v) .
\end{aligned}
$$

Since we can choose $\lambda>0$ satisfying

$$
\frac{k_{1}+k_{2}+2 p}{\lambda}+\frac{k_{1} k_{2}+K}{\lambda^{2}}<1,
$$

we receive $N_{3}$ is a contraction operator. Hence $N_{3}$ has a unique fixed point, that is a solution of the problem (14)-(17). The theorem is proven completely.

Remark 1. In ${ }^{15}$ Arara et al. studied the existence of fuzzy solutions of equation (1) with local conditions (2) and (3). However, these results base on some constraint conditions on the domain, such as condition $K a b<1$. These conditions are too strict for the domain $J_{a b}$ to satisfy if the Lipschitz constant $K$ is big enough. On the other hand, it depends on the large scale of the domain. To relax this restriction, in this paper we introduced new weighted metric $H_{\lambda}$ in the complete fuzzy metric space $C\left(J_{r}, E^{n}\right)$. Since then, we can extend some existence and uniqueness theorems to the whole domain without any constraints in the boundary of domain.

\section{Numerical examples}

In this section, we present some numerical examples showing the existence of fuzzy solutions for PHFDEs. Firstly by using the same strategy as Buckley-Feuring, we fuzzify the deterministic solution (see ${ }^{7,14}$ ) to build fuzzy solutions. After that, we use the continuity of Zadeh's extension principle and numerical simulation (see for more details in ${ }^{16}$ ) to show some graphical representations of the fuzzy solutions.

Example 1. Consider the following fuzzy PHFDEs

$$
U_{x y}+2(y U)_{y}=2 U_{(x, y)}\left(-\frac{1}{2},-\frac{1}{2}\right)+C,
$$

for $(x, y) \in J_{11}=[0,1] \times[0,1]$, with the local conditions

$$
U(x, 0)=C x+C, U(0, y)=C, U(0,0)=C,
$$

where $C$ is a fuzzy number in universal interval $I=[0, M], M>0$ and

$$
U(x, y)=C x y+C x+1,
$$

for $(x, y) \in \widetilde{J}_{1 / 2}=\left[-\frac{1}{2}, 1\right] \times\left[-\frac{1}{2}, 1\right] \backslash(0,1] \times(0,1]$.

From (26), we have

$$
F\left(x, y, U_{(x, y)}\right)=2 U\left(x-\frac{1}{2}, y-\frac{1}{2}\right)+C,
$$

thus

$$
\begin{aligned}
& d_{\infty}\left(F\left(x, y, U_{(x, y)}\right), F\left(x, y, V_{(x, y)}\right)\right) \\
& \quad \leqslant 2 d_{\infty}\left(U\left(x-\frac{1}{2}, y-\frac{1}{2}\right), V\left(x-\frac{1}{2}, y-\frac{1}{2}\right)\right) .
\end{aligned}
$$

It means that the Hypothesis $(H)$ is satisfied with $K=2$. Since $p(x, y)=-2 y$, we have

sup $|p(x, y)|=2$. So, from Theorem 3.2 we see $(x, y) \in[0,1]^{2}$

that, if we choose positive weighted number $\lambda$ satisfying

$$
\lambda^{2}-2 \lambda-2>0 \text { or } \lambda>1+\sqrt{3},
$$

then there exists a unique fuzzy solution $U$ of problem (26)-(28) in the weighted metric space $\left(C\left(J_{1 / 2}, E^{2}\right), H_{\lambda}\right)$.

The deterministic solution of the crisp PHFDEs corresponding to (26)-(28) is $u(x, y)=g(x, y, c)=$ $c x+c$. We now fuzzify this crisp solution to find fuzzy solution of fuzzy PHFDEs (26)-(28). To this end, let us denote $h(x, y, c)=c$. We apply the fuzzification in $c$, and supposed that the parametric form of corresponding fuzzy number $C$ is

$$
[C]^{\alpha}=\left[c_{1}(\alpha), c_{2}(\alpha)\right]
$$

where the sufficient conditions are

(a) $c_{1}(\alpha)$ is a bounded left continuous nondecreasing function with respect to $\alpha$.

(b) $c_{2}(\alpha)$ is a bounded left continuous nonincreasing function with respect to $\alpha$.

(c) $c_{1}(\alpha) \leqslant c_{2}(\alpha)$, for all $\alpha \in[0,1]$.

By using the extension principle, $H$ is computed from $h$ and $Y$ is computed from $g$. We will show that $Y$ is the fuzzy solution of this problem. Indeed, 
since all the partials of $h$ and $g$ with respect to $c$ are positive. We see that

$$
\begin{aligned}
{[H(x, y, C)]^{\alpha} } & =\left[H_{1}(x, y, \alpha), H_{2}(x, y, \alpha)\right] \\
& =\left[\min _{c \in[C]^{\alpha}}\{h(x, y, c)\}, \max _{c \in[C]^{\alpha}}\{h(x, y, c)\}\right] \\
& =\left[c_{1}(\alpha), c_{2}(\alpha)\right]
\end{aligned}
$$

satisfied conditions $(a)-(c)$. So $[H(x, y, C)]^{\alpha}$ is the $\alpha$-cuts of fuzzy number $C$. Similarly

$$
\begin{aligned}
{[Y(x, y, C)]^{\alpha} } & =\left[Y_{1}(x, y, \alpha), Y_{2}(x, y, \alpha)\right] \\
& =\left[\min _{c \in[C]^{\alpha}}\{g(x, y, c)\}, \max _{c \in[C]^{\alpha}}\{g(x, y, c)]\right. \\
& =\left[c_{1}(\alpha) x+c_{1}(\alpha), c_{2}(\alpha) x+c_{2}(\alpha)\right]
\end{aligned}
$$

are the $\alpha$-cuts of fuzzy number $C x+C$. Define differential operator

$\varphi\left(D_{x}, D_{y}\right) U(x, y)=U_{x y}+2(y U)_{y}-2 U_{(x, y)}\left(-\frac{1}{2},-\frac{1}{2}\right)$

and

$$
\begin{aligned}
S(x, y, \alpha)=\left[\varphi\left(D_{x}, D_{y}\right) Y_{1}(x, y, \alpha),\right. & \\
& \left.\varphi\left(D_{x}, D_{y}\right) Y_{2}(x, y, \alpha)\right] .
\end{aligned}
$$

We first check to see if $Y(x, y)$ is differentiable. We compute

$$
\left[\varphi\left(D_{x}, D_{y}\right) Y_{1}(x, y, \alpha), \varphi\left(D_{x}, D_{y}\right) Y_{2}(x, y, \alpha)\right]
$$

which equals to $\left[c_{1}(\alpha), c_{2}(\alpha)\right]$, which are the $\alpha$-cuts of fuzzy number $C$. Hence, $Y(x, y)$ is fuzzy differentiable. Because all partials of $h$ and $g$ with respect to $c$ are positive. And we easily see that

$$
\begin{aligned}
& Y_{1}(x, 0, \alpha)=c_{1}(\alpha) x+c_{1}(\alpha) \\
& Y_{2}(x, 0, \alpha)=c_{2}(\alpha) x+c_{2}(\alpha) \\
& Y_{1}(0, y, \alpha)=Y_{1}(0,0, \alpha)=c_{1}(\alpha) \\
& Y_{2}(0, y, \alpha)=Y_{2}(0,0, \alpha)=c_{2}(\alpha)
\end{aligned}
$$

which are all true. Therefore, $Y(x, y)$ is the fuzzy solution of (26)-(28) in the sense of Buckley and Feuring (see in ${ }^{7,23}$ ), which satisfies the boundary conditions. This solution may be written as

$$
U(x, y)=C x+C
$$

Now to have a figure of $\alpha$-cut of the solutions, we consider fuzzy number $C$ with membership function is Laplace function ${ }^{24}$

$$
C(t)=2\left(1+\exp \left(\frac{\pi|t-c|}{\sigma \sqrt{6}}\right)\right)^{-1}, t>0
$$

It is easily to find the $\alpha$-cuts of $C$ are

$$
[C]^{\alpha}=\left[c-\frac{\sigma \sqrt{6}}{\pi} \ln \left(\frac{2}{\alpha}-1\right), c+\frac{\sigma \sqrt{6}}{\pi} \ln \left(\frac{2}{\alpha}-1\right)\right],
$$

for $\alpha \in(0,1]$.

The continuity of extension principle states that the $\alpha$-cuts of $U(x, y)=C x+C((x, y)$ is fixed $)$ are

$$
\begin{aligned}
{[U(x, y)]^{\alpha}=\left[(x+1)\left[c-\frac{\sigma \sqrt{6}}{\pi} \ln \left(\frac{2}{\alpha}-1\right)\right],\right.} \\
\left.(x+1)\left[c+\frac{\sigma \sqrt{6}}{\pi} \ln \left(\frac{2}{\alpha}-1\right)\right]\right] .
\end{aligned}
$$

Obviously, the deterministic solution is the preferred solution $[U(x, y)]^{1}$, which means that it has membership degree 1 . So the membership function of $U(x, y)$ is

$$
U(x, y)(t)=2\left(1+\exp \left(\frac{\pi\left|\frac{t}{x+1}-c\right|}{\sigma \sqrt{6}}\right)\right)^{-1} .
$$

Figure 1 and 2 shows some graphs of membership functions of Laplace fuzzy numbers and $U(x, y)(t)$ at some fixed values of $(x, y) \in J_{11}$.

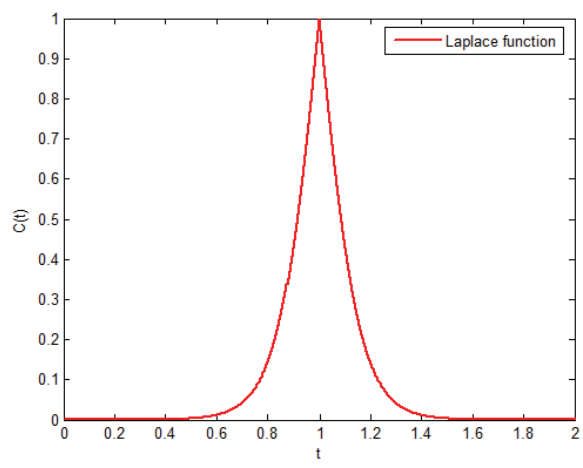

Fig. 1. The curve of Laplace fuzzy number. 
fuzzy PHFDEs

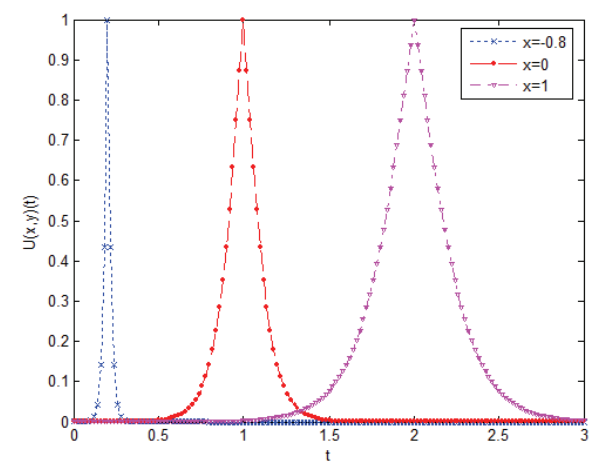

Fig. 2. The curve of fuzzy solution $U(x, y)$ at different values of $(x, y)$.

A simulation was carried out using numerical methods for the deterministic solution together with the continuity of Zadeh's extension principle. Figure 3 shows five concrete $\alpha$-cuts of fuzzy solutions. Concretely, the first and the fifth dark curves from the bottom to the top of the surface correspond to the lower bounded and the upper bounded solutions of $U(x, y)$. They are the curves of level set $[U(x, y)]^{0.0001}$, here we choose $\alpha=0.0001$, a small enough level number, for demonstrating upper-lower bounded solutions. The second and the fourth ones are the curves of level set $[U(x, y)]^{0.5}$, which are the medium membership degree of solutions. Finally, the dark curve in the center of the surface corresponds to the deterministic solution $[U(x, y)]^{1}=c(x+1)$.

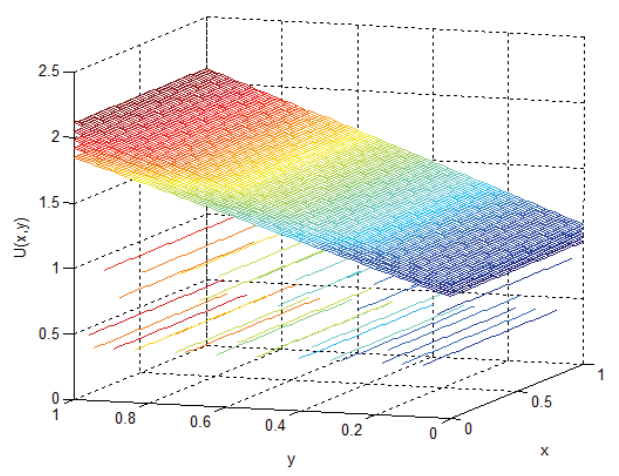

Fig. 3. Some $\alpha$-cuts of fuzzy PHFDEs (26)-(28). The dark curve in the center of the surface corresponds to the deterministic solution $[U(x, y)]^{1}$.

Example 2. In this example we consider following

$$
\begin{aligned}
& U_{x y}-\left(\exp \left(-\frac{1}{4}\right) U\right)_{y} \\
& =-U_{(x, y)}\left(\frac{-1}{4}, \frac{-1}{4}\right)+C_{1} \cos \left(x-\frac{1}{4}\right)+C_{3}
\end{aligned}
$$

with the boundary conditions are

$$
\begin{aligned}
& U(x, 0)+\int_{0}^{y} U(x, s) d s \\
& =\left(C_{1} \cos x+C_{3}\right)(y+1)+C_{2} \exp (y)
\end{aligned}
$$

and

$$
\begin{aligned}
& U(0, y)+\int_{0}^{x} U(t, y) d t \\
& =C_{1}(1+\sin x)+C_{2}(1+x) \exp (y)+C_{3}(1+x)
\end{aligned}
$$

where $(x, y) \in J_{\frac{\pi}{2} 1}:=\left[0, \frac{\pi}{2}\right] \times[0,1], C_{i}$ are fuzzy numbers in $I_{i}=\left[0, M_{i}\right], M_{i}>0(i=1,2,3)$ and the initial conditions is

$$
U(x, y)=C_{1} \cos [x(y+1)]+C_{2}\left(e^{y}+x y\right)+C_{3},
$$

here $(x, y) \in \widetilde{J}_{\frac{1}{4}}=\left[\frac{-1}{4}, \frac{\pi}{2}\right] \times\left[\frac{-1}{4}, 1\right] \backslash\left(0, \frac{\pi}{2}\right] \times(0,1]$.

Since

$F\left(x, y, U_{(x, y)}\right)=-U_{(x, y)}\left(\frac{-1}{4}, \frac{-1}{4}\right)+C_{1} \cos \left(x-\frac{1}{4}\right)+C_{3}$

satisfied

$$
\begin{aligned}
& d_{\infty}\left(F\left(x, y, U_{(x, y)}\right), F\left(x, y, V_{(x, y)}\right)\right) \\
& \quad \leqslant d_{\infty}\left(U_{(x, y)}\left(-\frac{1}{4},-\frac{1}{4}\right), V_{(x, y)}\left(-\frac{1}{4},-\frac{1}{4}\right)\right),
\end{aligned}
$$

for all $U, V \in C\left(J_{1 / 4}, E^{2}\right),(x, y) \in J_{\frac{\pi}{2} 1}$. It means that Hypothesis $(H)$ is satisfied with $K=1$. Due to $k_{1}=k_{2}=1, p=e^{-1 / 4}$, from inequality

$$
\frac{2+2 e^{-1 / 4}}{\lambda}+\frac{2}{\lambda^{2}}<1
$$

we imply that $\lambda>1+e^{-1 / 4}+\sqrt{3+2 e^{-1 / 4}+e^{-1 / 2}}$. Thus all the conditions of Theorem 4.1 are hold. And we conclude that there exists a unique fuzzy solution $U$ of problem (29)-(32) in the weighted metric space $\left(C\left(J_{1 / 4}, E^{n}\right), H_{\lambda}\right)$. 
Let $h(x, y, c)=c_{1} \cos \left(x-\frac{1}{4}\right)+c_{3}$, where $c=$ $\left(c_{1}, c_{2}, c_{3}\right)$. The solution of the crisp PHFDEs corresponding to (29)-(31) is $u(x, y)=g(x, y, c)=$ $c_{1} \cos x+c_{2} \exp (y)+c_{3}$, where $c_{i} \in I_{i},(x, y) \in J_{\frac{\pi}{2} 1}$. We apply the fuzzification in $c_{i}$, gained the parametric form of $C_{i}$

$$
\left[C_{i}\right]^{\alpha}=\left[c_{i 1}(\alpha), c_{i 2}(\alpha)\right], \alpha \in[0,1] .
$$

Let $[C]^{\alpha}=\Pi\left[C_{i}\right]^{\alpha}$. By using the extension principle, we compute $H$ from $h$ and $Y$ from $g$. After that, we will show $Y$ being fuzzy solution of this problem. Indeed, since the partials of $h$ and $g$ with respect to $c_{i}$ are positive

$$
\begin{aligned}
{[H(x, y, C)]^{\alpha} } & =\left[H_{1}(x, y, \alpha), H_{2}(x, y, \alpha)\right] \\
& =\left[\min _{c \in[C]^{\alpha}}\{h(x, y, c)\}, \max _{c \in[C]^{\alpha}}\{h(x, y, c)\}\right]
\end{aligned}
$$

which equals to

$$
\begin{aligned}
& {\left[c_{11}(\alpha) \cos \left(x-\frac{1}{4}\right)+c_{31}(\alpha),\right.} \\
& \left.\quad c_{12}(\alpha) \cos \left(x-\frac{1}{4}\right)+c_{32}(\alpha)\right]
\end{aligned}
$$

satisfied conditions $(a)-(c)$. So $[H(x, y, C)]^{\alpha}$ is the $\alpha$-cuts of fuzzy number $C_{1} \cos \left(x-\frac{1}{4}\right)+C_{3}$. Similarly

$$
\begin{aligned}
& {[Y(x, y, C)]^{\alpha}=\left[Y_{1}(x, y, \alpha), Y_{2}(x, y, \alpha)\right]} \\
& =\left[\min _{c \in[C]^{\alpha}}\{g(x, y, c)\}, \max _{c \in[C]^{\alpha}}\{g(x, y, c)]\right. \\
& =\left[c_{11}(\alpha) \cos x+c_{21}(\alpha) \exp (y)+c_{31}(\alpha),\right. \\
& \left.\quad c_{12}(\alpha) \cos x+c_{22}(\alpha) \exp (y)+c_{32}(\alpha)\right]
\end{aligned}
$$

is the $\alpha$-cuts of fuzzy number $C_{1} \cos x+$ $C_{2} \exp (y)+C_{3}$.

Define differential operator

$$
\begin{aligned}
\varphi\left(D_{x}, D_{y}\right) & U(x, y) \\
& =U_{x y}-\left(\exp \left(-\frac{1}{4}\right) U\right)_{y}+U\left(x-\frac{1}{4}, y-\frac{1}{4}\right)
\end{aligned}
$$

and

$$
\begin{array}{r}
S(x, y, \alpha)=\left[\varphi\left(D_{x}, D_{y}\right) Y_{1}(x, y, \alpha),\right. \\
\left.\varphi\left(D_{x}, D_{y}\right) Y_{2}(x, y, \alpha)\right] .
\end{array}
$$

We first check to see if $Y(x, y)$ is differentiable. We compute

$$
\left[\varphi\left(D_{x}, D_{y}\right) Y_{1}(x, y, \alpha), \varphi\left(D_{x}, D_{y}\right) Y_{2}(x, y, \alpha)\right]
$$

which equals to

$$
\begin{aligned}
& {\left[c_{11}(\alpha) \cos \left(x-\frac{1}{4}\right)+c_{31}(\alpha),\right.} \\
& \left.\quad c_{12}(\alpha) \cos \left(x-\frac{1}{4}\right)+c_{32}(\alpha)\right]
\end{aligned}
$$

which are $\alpha$-cut of fuzzy number $C_{1} \cos \left(x-\frac{1}{4}\right)+C_{3}$. Hence, $Y(x, y)$ is differentiable.

Because all partials of $h$ and $g$ with respect to $c_{i}$ are positive, $Y(x, y)$ is a fuzzy solution in the sense of Buckley and Feuring. The integral boundary conditions

$$
\begin{aligned}
& Y_{1}(x, 0, \alpha)+\int_{0}^{y} Y_{1}(x, s) d s \\
& =\left[C_{11}(\alpha) \cos x+C_{31}(\alpha)\right](y+1)+C_{21}(\alpha) \exp (y) \\
& Y_{2}(x, 0, \alpha)+\int_{0}^{y} Y_{2}(x, s) d s \\
& =\left[C_{12}(\alpha) \cos x+C_{32}(\alpha)\right](y+1)+C_{22}(\alpha) \exp (y \\
& Y_{1}(0, y, \alpha)+\int_{0}^{x} Y_{1}(t, y) d t \\
& \quad=C_{11}(\alpha)(1+\sin x)+C_{21}(\alpha)(1+x) \exp (y) \\
& \quad+C_{31}(\alpha)(1+x) \\
& \quad \\
& Y_{2}(0, y, \alpha)+\int_{0}^{x} Y_{2}(t, y) d t \\
& \quad=C_{12}(\alpha)(1+\sin x)+C_{22}(\alpha)(1+x) \exp (y) \\
& \quad+C_{32}(\alpha)(1+x)
\end{aligned}
$$

are all true. Therefore, $Y(x, y)$ is fuzzy solution which also satisfies the boundary conditions. This solution may be written as

$$
U(x, y)=C_{1} \cos x+C_{2} \exp (y)+C_{3} .
$$

By using the continuity of extension principle and Matlab simulation we will show some numerical fuzzy solutions of problem. 
Case 1. Firstly, we consider $C_{1}=C_{2}=C_{3}=C$, where $C$ is a Gaussian fuzzy number ${ }^{24}$

$$
C(t)=\exp \left(-2500(t-c)^{2}\right), t \in[0, \infty) .
$$

The $\alpha$-cuts of $C$ are

$$
\begin{aligned}
{[C]^{\alpha} } & =\left[c_{1}(\alpha), c_{2}(\alpha)\right] \\
& =\left[c-0.02 \sqrt{\ln \frac{1}{\alpha}}, c+0.02 \sqrt{\ln \frac{1}{\alpha}}\right]
\end{aligned}
$$

for $\alpha \in[0,1]$.

The continuity of Zadeh's extension principle states that fuzzy solution of (29) - (32) are

$$
\begin{aligned}
{[U(x, y)]^{\alpha}=} & {\left[\left(c-0.02 \sqrt{\ln \frac{1}{\alpha}}\right)(\cos x+\exp (y)+1),\right.} \\
& \left.\left(c+0.02 \sqrt{\ln \frac{1}{\alpha}}\right)(\cos x+\exp (y)+1)\right] .
\end{aligned}
$$

So we can convert this interval valued function into single valued function, that is the membership function of $U(x, y)$

$U(x, y)(t)=\exp \left(-2500\left[\frac{t}{\cos x+\exp (y)+1}-c\right]^{2}\right)$.

Figures 4 and 5 show some graph of membership functions of Gaussian fuzzy number and fuzzy solution $U(x, y)$ at different values of $(x, y)$.

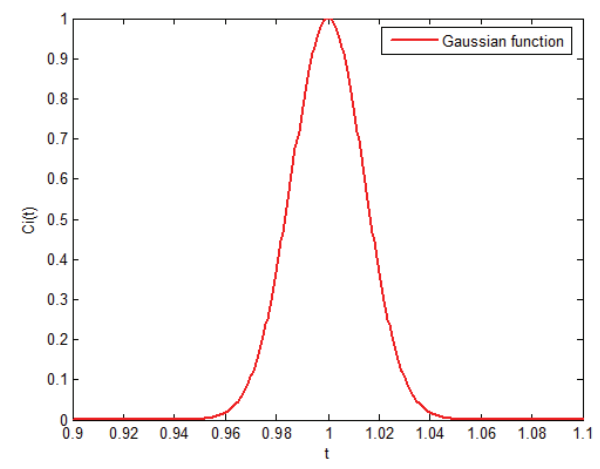

Fig. 4. The curve of Gaussian fuzzy number.

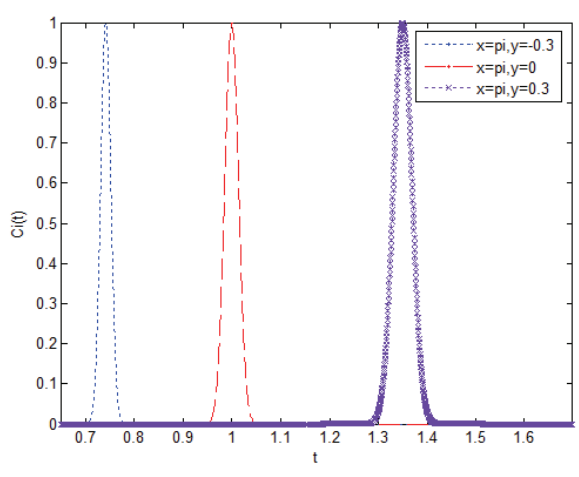

Fig. 5. The curve of fuzzy solutions $U(x, y)$ at different values of $(x, y)$.

Some simulations for three $\alpha$-cuts of fuzzy solution are shown in Figure 6. The dark curves from the bottom to the top of the surface correspond to the lower bounded, the deterministic (level set $\left.[U(x, y)]^{1}\right)$ and the upper bounded solutions of $U(x, y)$.

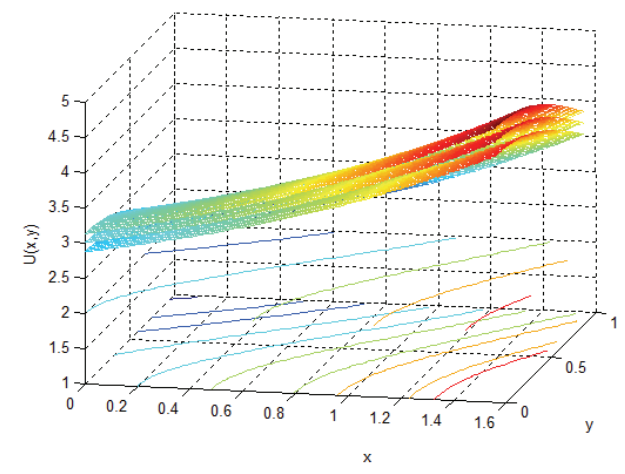

Fig. 6. The lower bounded, deterministic and upper bounded solutions of PHFDEs (29)-(32).

Figures 7 and 8 show some 2D graphs of some $\alpha$-cuts of fuzzy solution. The continuous line in the center corresponds to the deterministic solution. 


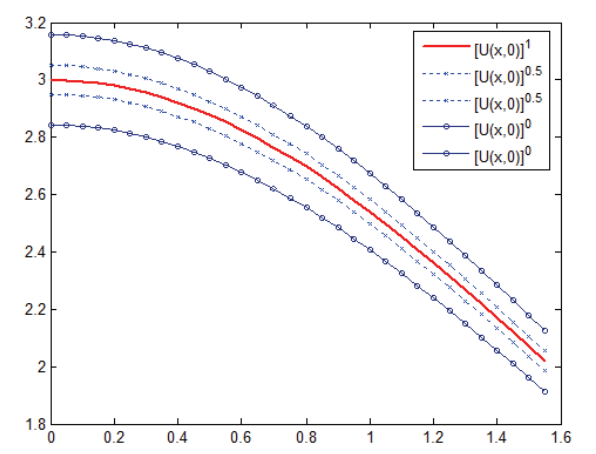

Fig. 7. The 2D graphs of some $\alpha$-cuts of fuzzy solution.

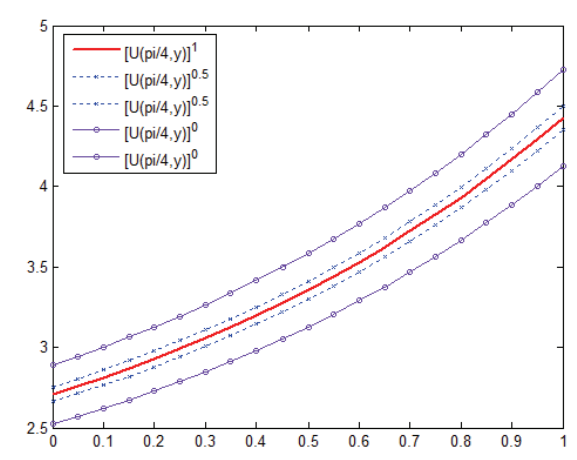

Fig. 8. The 2D graphs of some $\alpha$-cuts of fuzzy solution.

Case 2. In this part, we consider $C_{i}=$ $\left(a_{i}, c_{i}, b_{i}\right),(i=1,2,3)$ are three different triangular fuzzy numbers with membership functions in the form

$$
C_{i}(t)=\left\{\begin{array}{l}
\frac{t-a_{i}}{c_{i}-a_{i}} \text { if } a_{i} \leqslant t \leqslant c_{i} \\
\frac{b_{i}-t}{b_{i}-c_{i}} \text { if } c_{i} \leqslant t \leqslant b_{i} \\
0 \quad \text { otherwise. }
\end{array}\right.
$$

The $\alpha$-cuts of $C_{i}$ are

$$
\left[a_{i}+\left(c_{i}-a_{i}\right) \alpha, b_{i}-\left(b_{i}-c_{i}\right) \alpha\right] .
$$

Then the $\alpha$-cuts of fuzzy solution are

$$
\begin{aligned}
{[\cos x} & {\left[a_{1}+\left(c_{1}-a_{1}\right) \alpha\right]+\exp (y)\left[a_{2}+\left(c_{2}-a_{2}\right) \alpha\right] } \\
& +a_{3}+\left(c_{3}-a_{3}\right) \alpha, \cos x\left[b_{1}-\left(b_{1}-c_{1}\right) \alpha\right] \\
& \left.+\exp (y)\left[b_{2}-\left(b_{2}-c_{2}\right) \alpha\right]+b_{3}-\left(b_{3}-c_{3}\right) \alpha\right] .
\end{aligned}
$$

This is the parametric form of an triangular fuzzy number, so the membership fuzzy of fuzzy solution is

$$
\begin{gathered}
U(x, y)(t)=\left(a_{1} \cos x+a_{2} \exp (y)+a_{3}, c_{1} \cos x\right. \\
\left.+c_{2} \exp (y)+c_{3}, b_{1} \cos x+b_{2} \exp (y)+b_{3}\right)
\end{gathered}
$$

Figure 9 shows membership functions of $C_{1}=$ $(0,0.5,1), C_{2}=(0.5,1,2), C_{3}=(0.7,1.5,2)$ and $U(0,0)=C_{1}+C_{2}+C_{3}$.

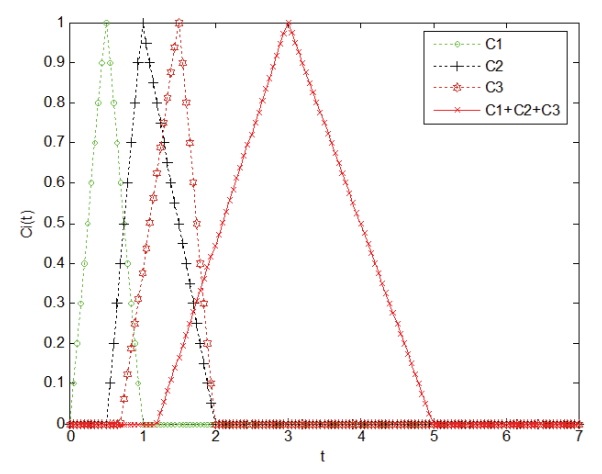

Fig. 9. The curves of some triangular fuzzy numbers.

Numerical simulations are used to obtain a graphical representation of the fuzzy solution. The surface of fuzzy solution is shown in figure 10 .

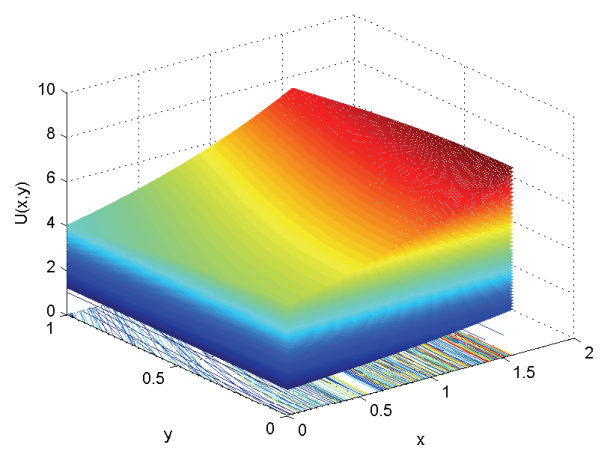

Fig. 10. The surface of fuzzy solution with triangular fuzzy numbers $C_{i}, i=1,2,3$.

\section{Conclusions}

The main ambition of this article has been to investigate the fuzzy solutions of some class of 
partial hyperbolic functional differential equations with local conditions and integral boundary conditions. Our study provides a theoretical foundation for many numerical solution methods and computational intelligence of some classes of fuzzy PDEs and ensures the consistency, stability and convergence of computational algorithms. We illustrate our results by some computational examples, in which we compute the fuzzy exact solutions of some fuzzy boundary problems with time delay. The next step in the direction proposed here is to study the fuzzy solution for the partial hyperbolic functional differential equations with the nonlocal conditions.

\section{Acknowledgments}

The authors are greatly indebted to Editor-inChiefs Prof. Martínez Lopez and anonymous reviewers for your comments and your valuable suggestions that improve the quality and clarity of the paper.

\section{References}

1. L.A. Zadeh, Fuzzy sets, Information and Control, 8 (1965), pp. 338-353.

2. P. Diamond, P.E. Kloeden, Metric spaces of fuzzy sets: Theory and Applications, World Scientific, Singapore, 1994.

3. V. Lakshmikantham and R.N. Mohapatra, Theory of fuzzy differential equations and inclusions, Taylor and Francis Publishers, London, 2003.

4. S.L. Chang, L.A. Zadeh, On fuzzy mapping and control, IEEE Transactions on Systems, Man, and Cybernetics, 2(1972), pp. 30-34.

5. D. Dubois and H. Prade, Towards fuzzy differential calculus, Part III, Fuzzy Sets and Systems, 8(1982), pp. 225-234.

6. M. Puri and D. Ralescu, Fuzzy random variables, Journal of Mathematical Analysis and Applications, 114(2)(1986), pp. 409-422.

7. J.J. Buckley and T. Feuring, Introduce to fuzzy partial differential equations, Fuzzy Sets and Systems, 105(1999), pp. 241-248.

8. O. Kaleva, Fuzzy differential equations, Fuzzy Sets and Systems, 24(1987), pp. 301-317.

9. S. Seikkala, On the fuzzy initial value problem, Fuzzy Sets and Systems, 24(1987), pp. 319-330.

10. Y. Chalco-Cano and H. Román-Flores, On new solutions of fuzzy differential equations, Chaos, Solitons \& Fractals, 38(1) (2008), pp. 112-119.
11. A.D.R. Choudary and T. Donchev, On Peano theorem for fuzzy differential equations, Fuzzy Sets and Systems, 177(2011), pp. 93-94.

12. J. Li, A. Zhao and J. Yan, The Cauchy problem of fuzzy differential equations under generalized differentiability, Fuzzy Sets and Systems, 200(2012), pp. 124.

13. R. Rodríguez-López, On the existence of solutions to periodic boundary value problems for fuzzy linear differential equations, Fuzzy Sets and Systems, 219 (2013), pp. 1-26.

14. T. Allahviranloo, S. Abbasbandy and H. Rouhparvar, The exact solutions of fuzzy wave-like equations with variable coefficients by a variational iteration method, Applied Soft Computing, 11(2011), pp. 2186-2192.

15. A. Arara, M. Benchohra, S.K. Ntouyas and A. Ouahab, Fuzzy solutions for hyperbolic partial differential equations, International Journal of Applied Mathematical Sciences, 2(2) (2005), pp. 181-195.

16. A.M. Bertone, R.M. Jafelice, L.C. de Barros and R.C. Bassanezi, On fuzzy solutions for partial differential equations, Fuzzy Sets and Systems, 219(2013), pp. 68-80.

17. H.V. Long, N.T.K. Son, N.T.M. Ha and L.H. Son, The existence and uniqueness of fuzzy solutions for hyperbolic partial differential equations, Fuzzy Optimization and Decision Making (2014), DOI 10.1007/s10700-014-9186-0.

18. Y.Y. Chen, Y.T. Chang and B. S. Chen, Fuzzy solutions to partial differential equations: Adaptive Approach, IEEE Transactions on Fuzzy Systems, 17(1) (2009), pp. 116-127.

19. F. Hartung, Differentiability of solutions with respect to parameters in neutral differential equations with state-dependent delays, Journal of Mathematical Analysis and Applications, 324(1)(2006), pp. 504524.

20. S. Abbas, M. Benchohra and G.M. N'Guérékata, Topics in fractional differential equations, Springer, Berlin, Heidelberg, New York, Hong Kong, London, Milan, Paris, Tokyo, 2012.

21. E. Hernandez, R. Sakthivel, S.T. Aki, Existence results for impulsive evolution differential equations with state-dependent delay, Electronic Journal of Differential Equations, 2008(2008), pp.1-11.

22. B. Ahmad, A. Alsaedi, and B. S. Alghamdi, Analytic approximation of solutions of the forced Duffing equation with integral boundary conditions, Nonlinear Analysis: Real World Applications, 9(4) (2008), pp. 1727-1740.

23. J.J. Buckley and T. Feuring, Fuzzy differential equations, Fuzzy Sets and Systems, 110 (2000), pp. 43-54.

24. H.V. Long, A note on the rates of uniform approximation of fuzzy systems, International Journal of Computational Intelligence Systems, 4(4)(2011), pp. 712727. 